\title{
ARTICLE
}

Cellular and Molecular Biology

\section{The HDAC6 inhibitor C1A modulates autophagy substrates in diverse cancer cells and induces cell death}

\author{
Maciej Kaliszczak ${ }^{1,3}$, Erich van Hechanova ${ }^{1,4}$, Yunqing $\mathrm{Li}^{1}$, Hibah Alsadah ${ }^{2}$, Katarzyna Parzych ${ }^{2}$, Holger W. Auner ${ }^{2}$ and Eric O. Aboagye ${ }^{1}$
}

BACKGROUND: Cytosolic deacetylase histone deacetylase 6 (HDAC6) is involved in the autophagy degradation pathway of malformed proteins, an important survival mechanism in cancer cells. We evaluated modulation of autophagy-related proteins and cell death by the HDAC6-selective inhibitor C1A.

METHODS: Autophagy substrates (light chain-3 (LC-3) and p62 proteins) and endoplasmic reticulum (ER) stress phenotype were determined. Caspase-3/7 activation and cellular proliferation assays were used to assess consequences of autophagy modulation. RESULTS: C1A potently resolved autophagy substrates induced by 3-methyladenine and chloroquine. The mechanism of autophagy inhibition by HDAC6 genetic knockout or C1A treatment was consistent with abrogation of autophagosome-lysosome fusion, and decrease of Myc protein. C1A alone or combined with the proteasome inhibitor, bortezomib, enhanced cell death in malignant cells, demonstrating the complementary roles of the proteasome and autophagy pathways for clearing malformed proteins. Myc-positive neuroblastoma, KRAS-positive colorectal cancer and multiple myeloma cells showed marked cell growth inhibition in response to HDAC6 inhibitors. Finally, growth of neuroblastoma xenografts was arrested in vivo by single agent C1A, while combination with bortezomib slowed the growth of colorectal cancer xenografts.

CONCLUSIONS: C1A resolves autophagy substrates in malignant cells and induces cell death, warranting its use for in vivo preclinical autophagy research.

British Journal of Cancer (2018) 119:1278-1287; https://doi.org/10.1038/s41416-018-0232-5

\section{INTRODUCTION}

Autophagy is a process for clearing malformed, damaged or superfluous proteins within the intracellular compartment into autophagosomes for delivery to lysosomes for degradation and recycling (Supplementary Fig. 1). While the context-specific role of autophagy in cancer is still debated, it is recognised that autophagy can serve two key functions: a tumour-suppressive function through elimination of oncogenic proteins, and perhaps for established cancer, a tumour-promoting function via recycling of metabolites to maintain mitochondrial functionality. ${ }^{1}$ It is the therapeutic role of autophagy-targeting drugs in cancer that is receiving recent attention due to the potential of such therapies to induce apoptosis or by-pass apoptosis defects to induce other forms of cytotoxicity. ${ }^{2}$ Enhanced autophagy can ensue following chemotherapy, and inhibition of autophagy under such conditions can lead to increased cell death as a cellular response to avoid accumulation of toxic proteins. ${ }^{3,4}$ Another important protein homeostasis mechanism in cells is governed by the proteasome. Proteasome inhibitors including bortezomib and carfilzomib are used clinically in multiple myeloma and selected other B cell malignancies, and are thought to exert their anti-tumour effects by triggering the accumulation of toxic misfolded proteins. ${ }^{5}$ Autophagy is widely thought to contribute to proteasome inhibitor resistance in myeloma by providing an alternative pathway for clearance of dysfunctional proteins. Cells can also curtail further accumulation of dysfunctional proteins through decreased protein translation via the unfolded protein response (UPR). The UPR is a highly conserved pathway that operates to prevent or correct a cellular phenotype termed endoplasmic reticulum (ER) stress via an adaptive response through a specific gene transcription programme; this response and autophagy are thought to control cell viability in cells where abnormal protein homeostasis persists. ${ }^{6}$

Unfortunately, to date, we have available only a limited number of inhibitors to study autophagy in vitro and in vivo. The antimalarial agent chloroquine (CQ) that blocks lysosome acidification (Supplementary Fig. 1) has been evaluated pre-clinically and in patients alone and in combination with chemotherapy for autophagy inhibition. ${ }^{7-9}$ These studies have demonstrated that it is difficult to achieve robust autophagy inhibition at tolerable dose levels of CQ. Antagonists of the phosphoinositide 3-kinasemammalian target of rapamycin pathway have also been tested as autophagy inhibitors. In particular, 3-methyladenine (3-MA; Supplementary Fig. 1) and 3-MA derivatives with improved solubility have been tested and show activity in vitro. ${ }^{10}$ Lastly, histone deacetylase 6 (HDAC6) inhibitors and pan-HDAC inhibitors

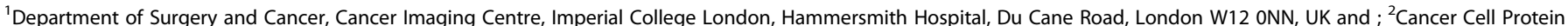

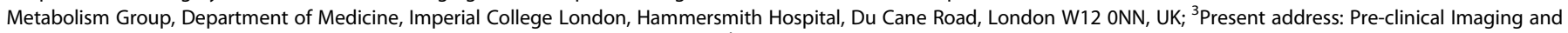

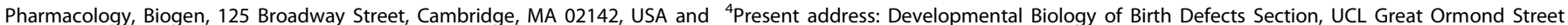
Institute of Child Health, University College London, 30 Guilford Street, London WC1N 1EH, UK

Correspondence: Eric O. Aboagye (eric.aboagye@imperial.ac.uk)

Received: 17 November 2017 Revised: 18 July 2018 Accepted: 25 July 2018

Published online: 15 October 2018 
have been evaluated as autophagy inhibitors. HDAC6 is involved in ubiquitin-dependent or ubiquitin-independent protein aggregate formation, as well as their clearance via autophagy. ${ }^{11-14}$ HDAC6, in association with the dynein motor complex, recruits and transports misfolded polyubiquitinated proteins via the microtubule network to aggresomes/autophagosomes for subsequent degradation by lysosomes. ${ }^{13,15}$ Pan-HDAC inhibitors combined with bortezomib are potent in resistant cancers due to the complementary roles of the autophagy and proteasome pathways in protein recycling; however, such combination is poorly tolerated clinically. ${ }^{16}$ It should be noted, however, that not all HDAC6 inhibitors can modulate autophagy. The lack of activity may be related to pharmacokinetics; the clinical HDAC6 candidate ricolinostat (ACY-1215) was recently reported to lack sufficient serum concentration to directly induce anti-multiple myeloma activity in vivo. ${ }^{17}$ However, at the cellular level, the HDAC6 inhibitor HPOB, while possessing potent HDAC6 deacetylase activity, does not modulate autophagy. ${ }^{17,18}$

We have previously described a selective HDAC6 inhibitor, C1A, with good pharmacokinetics and in vivo potency in solid tumour xenografts. ${ }^{19}$ In this work, we evaluated the effect of C1A on autophagy and cell death in cancer subtypes presumed to be susceptible to autophagy inhibition (including cells harbouring mutant KRAS, Myc protein, or cells that produce high levels of immunoglogulin and are dependent on efficient clearance of cytotoxic misfolded protein aggregates for survival). ${ }^{1,20,21}$

\section{MATERIALS AND METHODS}

Compounds

C1A was synthesised as previously described. ${ }^{19}$ Suberoylanilide hydroxamic acid (SAHA) and tubastatin A were purchased from Cayman Chemical (Ann Arbor, MI, USA). 3-MA and chloroquine were from Sigma (St Louis, MO, USA). Bortezomib was from EMD Millipore (Nottingham, UK). ACY-1215 was purchased from Selleckchem (Munich, Germany).

\section{Antibodies}

Antibodies against active cleaved caspase-3, C-Myc, HDAC6, LC3B and $\mathrm{N}-\mathrm{Myc}$, acetyltubulin and tubulin, acetyl-H3 and $\mathrm{H} 3$ were from Cell Signalling (Beverly, MA, USA). Anti- $\beta$-actin was from Abcam (Cambridge, UK).

\section{Cells}

Colorectal cancer HCT116 cells were obtained from ATCC. Osteosarcoma U2OS cells transfected with light chain-3B (LC3B) were a gift from Prof. Michael Seckl (Imperial College London, London, UK). Mouse embryonic fibroblasts (MEFs) proficient (WT) and deficient $(\mathrm{KO})$ in HDAC6 were kindly provided by Prof. TsoPang Yao (Duke University, Durham, NC, USA). We have previously characterised these cells. ${ }^{22}$ Multiple myeloma cells were obtained from the American Type Culture Collection or Deutsche Sammlung von Mikroorganismen und Zellkulturen. Neuroblastoma cells were a gift from Prof. Louis Chelser (The Institute for Cancer Research, London, UK). The Tet21/N cells were generated by stable transduction of SHEP cells with a Tet-off (tetracycline withdrawalinducible) N-Myc expression construct. ${ }^{23}$ TGR-1 (Myc+/+, Myc-/and $M y c-/-$ wtMyc) rat fibroblast cell lines were kindly provided by Prof. Prochownik (University of Pittsburgh School of Medicine, Pittsburgh, PA, USA). These cells have previously been characterised for Myc expression. ${ }^{24}$ All cells were passaged in our laboratory for fewer than 6 months on receipt and were tested mycoplasma free.

\section{Immunofluorescence and p62 ELISA}

Exponentially growing HCT116 cells and osteosarcoma U2OS-LC3GFP cells were seeded in chamber slides (Sigma) on day 1 . The cells were treated on day 2 with different compounds for $24 \mathrm{~h}$ and stained by immunofluorescence as described elsewhere. ${ }^{25}$ The mCherry-GFP-LC3 construct was a generous gift from Dr. Terje Johansen (University of Troms $\emptyset$, Troms $\varnothing$, Norway). MEFs were transfected with the plasmid using Lipofectamine 2000 (Invitrogen, Thermo Fisher, Waltham, MA, USA) for $24 \mathrm{~h}$ before treatment with C1A. Cells were subsequently fixed with $4 \%$ paraformaldehyde, washed and visualised with a Olympus BX51 microscope (Olympus UK Ltd, London, UK). Positive cells were counted using the ImageJ software ( $\mathrm{NIH}$, Bethesda, MD, USA) and expressed as a percentage of total cells counted. Autophagy markers p62 were determined by enzyme-linked immunosorbent assay (ELISA) (kit from Enzo life Sciences, Farmingdale, NY, USA).

ER stress quantitation by real-time PCR

KELLY cells were treated continuously with reagents or vehicle for 24 or $72 \mathrm{~h}$. At the end of the incubation period both adherent and detached cells were pelleted, washed with phosphate-buffered saline and frozen at $-80^{\circ} \mathrm{C}$ prior to analysis. Transcripts were analysed after reverse transcription by real-time quantitative PCR as previously described. ${ }^{26}$ Results are shown as fold change in mRNA expression of the gene of interest, relative to GAPDH compared to the relevant vehicle-treated control condition.

\section{Caspase-3/7 assay}

Caspase-3/7 activity was determined using Promega's caspase-3/7 assay according to the manufacturer's instructions (Promega, Madison, WI, USA). Briefly, cells were transferred in a white opaque 96-well plate, incubated for $1 \mathrm{~h}$ with Caspase-Glo reagent and the enzymatic activity of caspase-3/7 was measured using a TopCount NXT microplate luminescence counter (PerkinElmer, Waltham, MA, USA). To enable normalisation of data to total cellular protein content, the sulforhodamine B (SRB) assay was performed in parallel for all samples. ${ }^{27}$

\section{ATPlite measurement assay}

Suspension cells were seeded into white, clear-bottom 96-well plates for $24 \mathrm{~h}$ and subsequently treated with C1A, ACY-1215, bortezomib alone or in combination for $24 \mathrm{~h}$. One hundred microliters of ATPlite luminescence (PerkinElmer) reagent was added and luminescence was measured using a TopCount NXT microplate luminescence counter (PerkinElmer).

Tumour xenografts

HCT116 $\left(5 \times 10^{6}\right)$ and KELLY $(7.5 \times 10)$ cells were injected subcutaneously in 100 and $150 \mu \mathrm{L}$ volumes, respectively, into the flank of female nu/nu-BALB/c athymic nude mice (Harlan, UK). Tumour measurements were performed every day and volumes were calculated using the formula [length $(\mathrm{mm})] \times[$ width $(\mathrm{mm})] \times$ [depth $(\mathrm{mm})] \times \pi / 6$. The animals were randomised, and when tumours had reached a volume of $50-100 \mathrm{~mm}^{3}$ animals were entered into the different treatment groups and treatment with different compounds was initiated (C1A, bortezomib alone or in combination). Animals were treated intraperitoneally ( $\mathrm{C} 1 \mathrm{~A}$ and bortezomib) for a total of 14 days. Both compounds were dissolved in dimethyl sulfoxide (DMSO) (10\%) and $0.9 \%$ saline. Throughout the 14-day treatment period, animal weights and tumour volumes were determined each day. All animal experiments were done by licensed investigators in accordance with the United Kingdom Home Office Guidance on the Operation of the Animal (Scientific Procedures) Act 1986 (HMSO, London, UK, 1990) and within guidelines set out by the United Kingdom National Cancer Research Institute Committee on Welfare of Animals in Cancer Research. ${ }^{28}$

Cell viability and cytotoxicity assays

Drug concentrations that inhibited $50 \%$ of cell growth $\left(\mathrm{GI}_{50}\right)$ for adherent cell lines were determined using an SRB technique. All cell lines were treated for $24 \mathrm{~h}$ on day 2 and allowed to grow for 
an additional 3 days. Optical densities were measured at $540 \mathrm{~nm}$ with a Multiskan EX photometer from Thermo Electron Corporation using the Ascent Software version 2.6 (Thermo Labsystems Oy, Vantaa, Finland). Growth inhibition curves were plotted as the percentage of control cells and $\mathrm{GI}_{50}$ values were determined by the GraphPad Prism 5 Software (San Diego, CA, USA) by fitting a sigmoidal curve with variable slope.

\section{Immunoblotting}

Cells and tumour tissue samples were prepared and subjected to western blotting as previously described. ${ }^{29}$ All primary antibodies were used at 1/1000 apart from anti- $\beta$-actin, which was diluted at $1: 10,000$. Anti-horseradish peroxidase-conjugated antimouse, antirabbit and anti-rat lgG secondary antibodies were used at a concentration of 1:2000. Densitometry data from western blot experiments were generated using the ImageJ software (NIH, USA) by measuring grey intensities of lanes corresponding to the protein of interest relative to the intensity of the lane corresponding to the loading control. Data are expressed as a fold change compared to control. It is indeed possible that some densitometry data (fold increase) were overestimated in cases where control values were nearly undetectable. This method is semi-quantitative and absolute data should indeed be interpreted with caution. Where available (e.g. measurements of p62) quantitative ELISA was used.
Statistical analyses

$T$ test or one-way analysis of variance with Dunnett's multiple comparisons test was used for analyses, which were performed using the GraphPad prism software (GraphPad, La Jolla, CA, USA), and $P$ values $<0.05$ using a $95 \%$ confidence interval were considered significant. Data are reported as mean \pm s.e.m. of at least three independent experiments, unless otherwise stated. ${ }^{*} P<0.05,{ }^{* *} P<0.005$ and ${ }^{* * *} P<0.0001$ were considered to be statistically significant; NS, not significant.

\section{RESULTS}

HDAC6 inhibition by C1A modulates autophagy

We induced autophagy and assessed changes in autophagy substrates under complete growth media conditions (quality control autophagy) following C1A treatment $(10 \mu \mathrm{M}, 24 \mathrm{~h})$. Treatment of colorectal cancer HCT116 cells (KRAS exon 2: G13D heterozygously mutated allele) with 3-MA ( $5 \mathrm{mM}, 24 \mathrm{~h})$ increased microtubule-associated protein-1 LC3 that is localised on autophagosomal membrane during autophagy (Fig. 1a). ${ }^{30,31}$ LC3 puncta determined by immunofluorescence (as green fluorescence using anti-LC3B antibody, D11) increased by 13-fold following 3-MA, an effect that was abrogated following co-incubation with $\mathrm{C} 1 \mathrm{~A}$. To confirm specificity of the process, we repeated the experiments in osteosarcoma U20S-LC3-GFP cells; the LC3-GFP fused protein is a
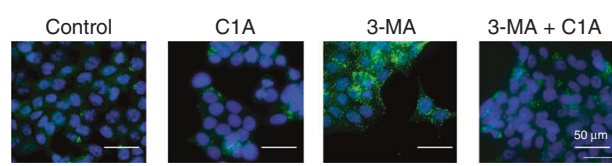

b
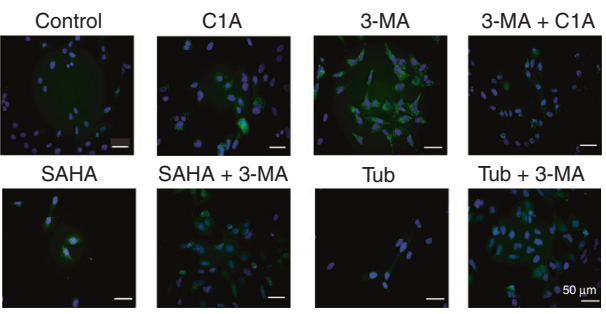
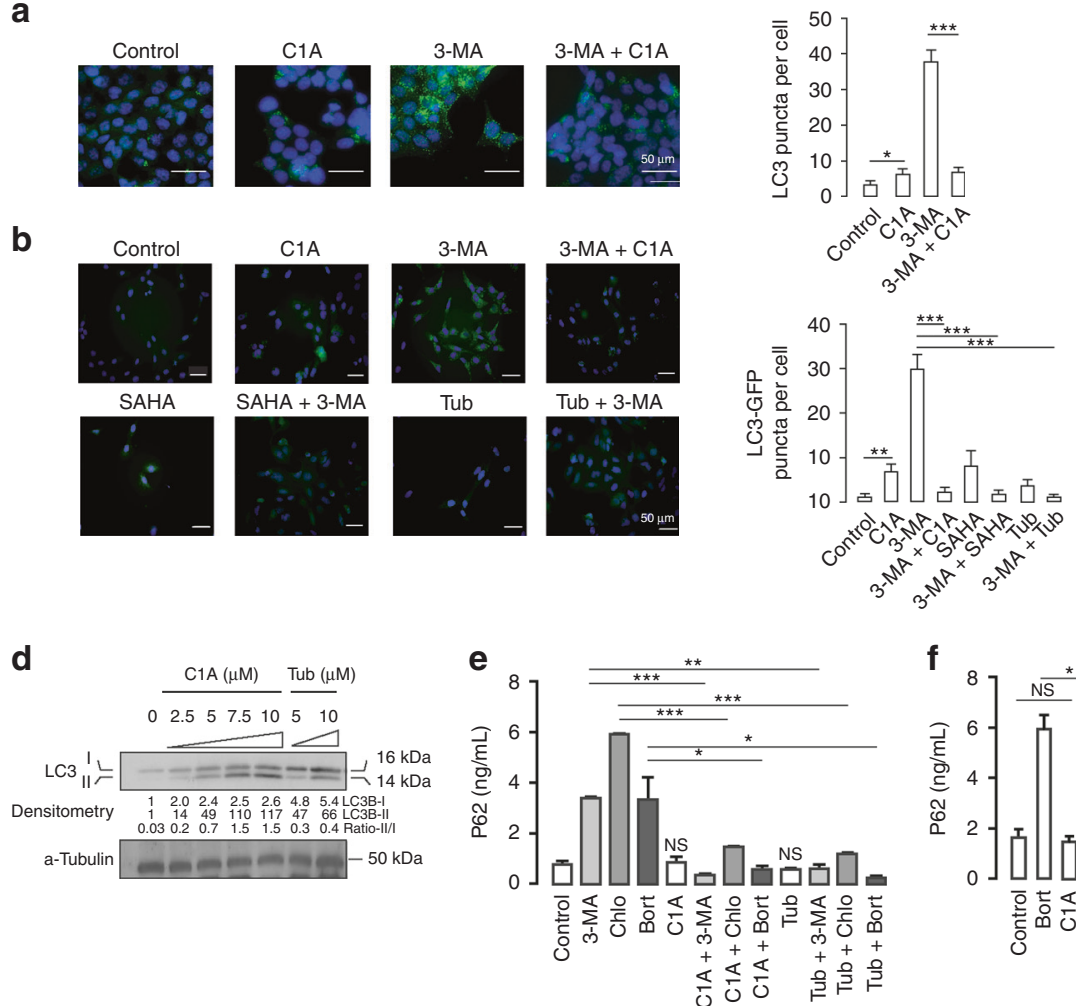

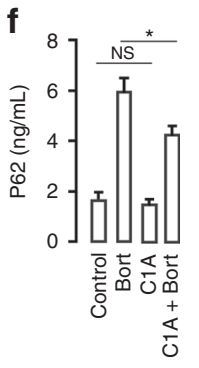

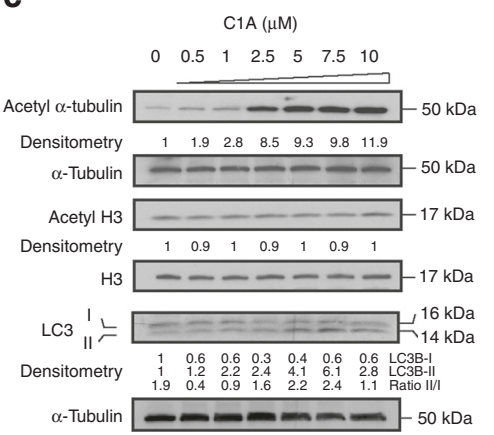

g

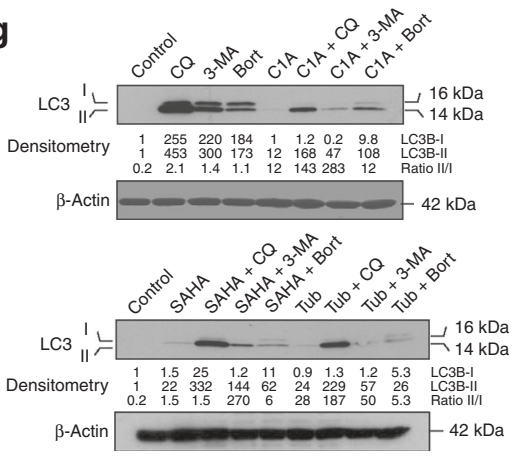

Fig. 1 C1A impairs autophagic maturation in colorectal cancer HCT116 cells and osteosarcoma U2OS-LC3-GFP cells. a, b Effect of C1A (10 $\mu$ M) on 3-MA ( $5 \mathrm{mM} ; 24 \mathrm{~h}$ ) induced increase of LC3 puncta (green) detected by immunofluorescence in HCT116 cells (a) and in U2OS-LC3-GFP cells (b). DNA (blue) was stained with DAPI. Treatment with SAHA and tubastatin A (Tub) are shown for comparison. Bar $=50 \mu m$ in each image. Summary data for counted puncta are shown next to the images; $>100$ cells were scored for each sample $(n=3)$. c, d Western blots of LC3 following $24 \mathrm{~h}$ treatment with C1A in HCT116 cells (c) and U2OS-LC3-GFP cells, stably transfected with LC3-GFP (d). The lower band corresponds to LC3B-II marker of autophagosome formation. Treatment with tubastatin A is shown for comparison. Acetylation of $\alpha$-tubulin was used as a marker of HDAC6 inhibition (vs. acetylation of histone H3, HDAC class I inhibition). ¥Note that absolute LC3-II/LC3I ratios are presented. e Levels of p62, a marker of autophagosome formation, as evaluated by ELISA in HCT116 cells. f Effect of C1A on bortezomibinduced increase of p62 levels in U2OS-LC3-GFP cells as determined by ELISA. g Effect of C1A on LC3 induction by autophagy tool compounds. HCT116 cells were treated with 3-MA $(5 \mathrm{mM})$, chloroquine (CQ- $50 \mu \mathrm{M})$, bortezomib (Bort, $5 \mathrm{nM}), \mathrm{SAHA}(10 \mu \mathrm{M})$, tubastatin A $(10 \mu \mathrm{M})$ and $\mathrm{C} 1 \mathrm{~A}(10 \mu \mathrm{M})$ alone or in combination for $24 \mathrm{~h}$ and assessed by western blot. Blots are representative of three independent experiments. Results are representative of three independent experiments performed in triplicate (mean \pm SEM). NS, not significant compared to control. ${ }^{*} P<0.05,{ }^{* *} P<0.005,{ }^{* * *} P<0.0001$ 
often used to detect autophagy through increased cytoplasmic GFP puncta. Again, C1A resolved GFP puncta induced by 3-MA (Fig. 1b). The pan-HDAC inhibitor SAHA $(10 \mu \mathrm{M}, 24 \mathrm{~h})$ and the HDAC6-selective tool compound, tubastatin A (10 $\mu \mathrm{M}, 24 \mathrm{~h})$, also resolved 3-MA-induced autophagy under similar conditions. Small increases in LC3 were seen by western blot at concentrations of C1A alone that affected HDAC6 catalytic function as demonstrated by acetylation of acetyl-a-tubulin but not acetyl-H3 (Fig. 1c, d); in this case, LC3I degradation that would be suggestive of enhanced 'autophagic flux' was not observed. Finally, changes in p62 expression can serve as a useful marker for the induction of autophagy. Increased p62 level can indeed represent a blockade of autophagy, and conversely, activation could be demonstrated by decreases in p62 levels. ${ }^{32,33}$ Notably, however, p62 can also be transcriptionally regulated by autophagy. ${ }^{34}$ Under the short-term treatments used here, C1 A and tubastatin A suppressed increases in 3-MA-induced, chloroquine-induced or bortezomib-induced p62 expression determined by ELISA (Fig. 1e, f).

Western blot analysis of LC3 was in keeping with the p62 assessment by ELISA (Fig. 1g). From the foregoing, it appears that C1A by itself mildly induces expression of LC3, but more importantly, is potent at resolving LC3 induced by 3-MA.

Given the published role of HDAC6 in transporting malformed proteins along dynein motors to the lysosomes, we determined whether C1A suppressed autophagosome fusion with lysosomes using a tandem tagged LC3-GFP-mCherry probe previously described by Ganley et al. ${ }^{35}$

While the low pH of the lysosome quenches GFP fluorescence, both GFP (green) and mCherry (red) fluoresce under the nearneutral $\mathrm{pH}$ outside the lysosome (Fig. 2). We found that MEFs transfected with the probe under complete media conditions show mainly mCherry fluorescence consistent with autophagosome fusion with lysosomes and GFP quenching. In contrast, MEFs in which HDAC6 has been genetically knocked out showed green and yellow fluorescence consistent with inhibition of fusion and this outcome was phenocopied by $\mathrm{C} 1 \mathrm{~A}$ treatment, demonstrating that $\mathrm{C} 1 \mathrm{~A}$ prevents autophagosome fusion with lysosomes.

C1A synergises with bortezomib in vitro and slows tumour growth in vivo

Towards clinical application, the pan-HDAC inhibitor, vorinostat (SAHA), has been shown to synergise with bortezomib to induce apoptosis in colorectal cancer cells via an autophagic mechanism. $^{36}$ Surmising that this cytotoxicity occurred via an HDAC6 function, we investigated the ability of C1A to induce caspase-3/7dependent cell death consequent to bortezomib-enhanced autophagy flux. C1A or bortezomib single treatment increased caspase-3/7 activity in HCT116 colorectal cells by 6-fold or 7-fold, respectively, over control. In contrast, the combination of $\mathrm{C} 1 \mathrm{~A}$ and bortezomib synergistically increased caspase-3/7 activity an order of magnitude higher (Fig. 3a). In addition, tubastatin A similarly synergised with bortezomib in the colon cancer cells. Bortezomib is approved for use in multiple myeloma and the HDAC6-selective inhibitor, ACY-1215, was shown to synergise with bortezomib in MM.1S and RPMI8226 myeloma cells, ${ }^{37}$ and as opposed to single agent showed $37 \%$ clinical response rate in multiple myeloma patients. ${ }^{38}$ In this report, we show that either C1A or ACY-1215 at equimolar concentrations synergises with bortezomib to induce caspase-3/7 activity in OPM-2 multiple myeloma cells (Fig. 3b). At the low micromolar concentrations of the drugs, near-complete loss of ATP accompanied the increase in caspase-3/7 activation (Fig. 3c), perhaps indicating a mechanism by which these compounds affect cell death, although this aspect needs further studies. Tubastatin A has limited activity in vivo due to poor pharmacokinetics. The potent and sustained activity of $\mathrm{C} 1 \mathrm{~A}$ relative to ACY-1215 under 'pulsed treatment-drug washout' conditions that mimic in vivo varying drug concentrations (Supplementary Fig. 2), and its demonstrated activity in vivo in solid tumours, makes C1A an ideal tool compound. ${ }^{19,22}$

HCT116 colon cancer cells reproducibly form tumours in immune-deficient mice and we have previously determined the efficacy of C1A in this model in vivo. ${ }^{19,22}$ From the foregoing, we hypothesised that $\mathrm{C} 1 \mathrm{~A}$ will potentiate the effect of bortezomib in HCT116 xenografts. Bortezomib was minimally effective in HCT116 xenografts (Fig. 3d). C1A slowed tumour growth and the combination of $\mathrm{C} 1 \mathrm{~A}$ and bortezomib was more potent than either drug alone (Fig. 3d). The combination was well tolerated in vivo with no significant change in body weight of mice (Fig. 3e).

\section{Exploring single agent activity of $\mathrm{C} 1 \mathrm{~A}$ in cell lineages with high Myc expression}

While the autophagy-modulating effects of different drug combinations have been reported, a deficiency in our current understanding of how to use HDAC6-selective inhibitors is the genetic basis to select patient populations that would be enriched for therapeutic response. KRAS-driven tumours and B cell lineage tumours, such as multiple myeloma (dependent on clearing high levels of immunoglobulin), have been suggested (vide supra). ${ }^{1,20}$ It would appear, however, that malignant cells with high expression of an oncogene that drives high levels of protein synthesis would be a candidate as cells generally lack efficient mechanisms to curtail superfluous protein synthesis and are thus dependent on
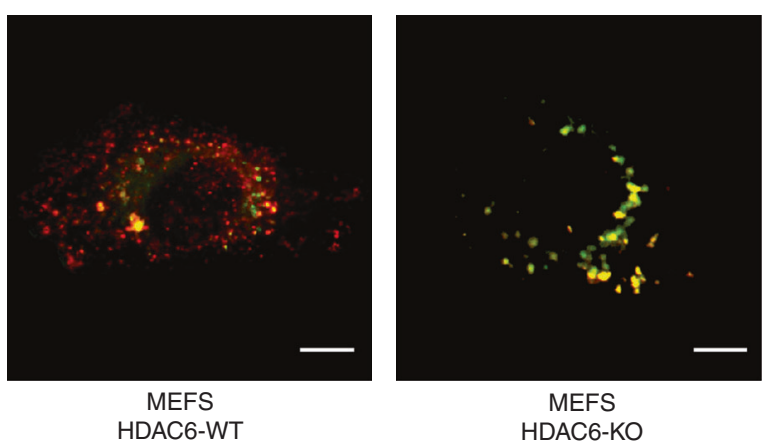

HDAC6-WT

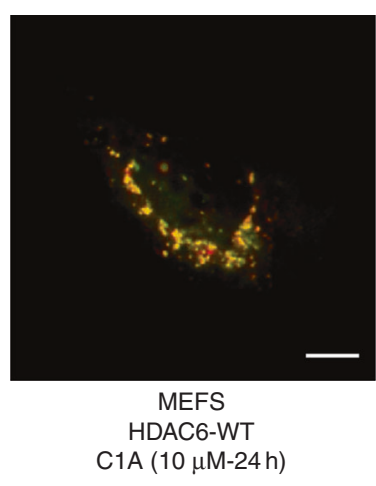
. 

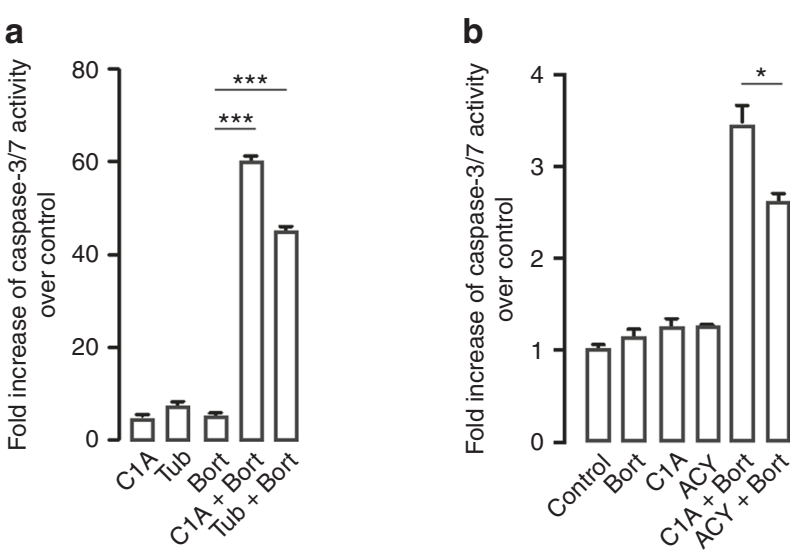

d

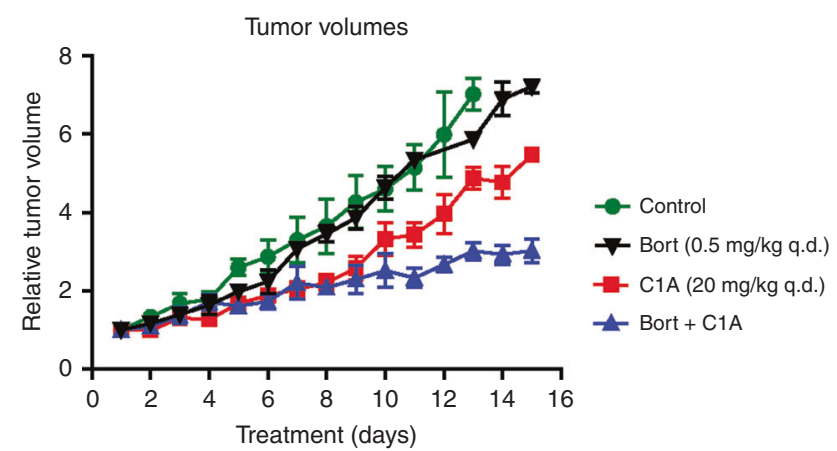

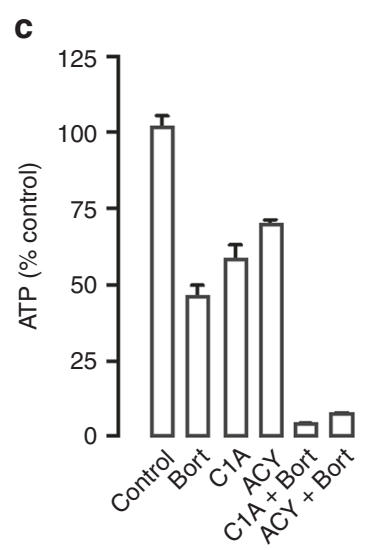

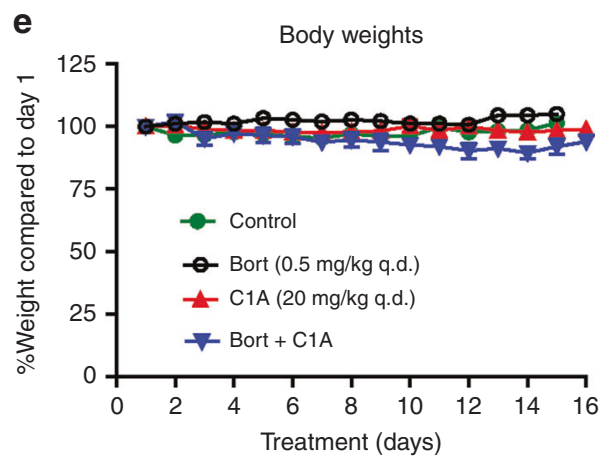

Fig. 3 HDAC6 inhibitor C1A synergises with proteasome inhibitor in vitro and in vivo. a Effect of $24 \mathrm{~h}$ treatment with bortezomib (Bort, $5 \mathrm{nM}$ ) alone or in combination with $\mathrm{C} 1 \mathrm{~A}(10 \mu \mathrm{M})$ or tubastatin $\mathrm{A}(\mathrm{Tub}, 10 \mu \mathrm{M})$ on caspase-3/7 activity in $\mathrm{HCT} 116 \mathrm{cells}$. b, $\mathbf{c}$ Effect of $9 \mathrm{~h}$ treatment with bortezomib (Bort, $5 \mathrm{nM}$ ) alone or in combination with C1A $(2 \mu \mathrm{M})$ or ACY-1215 (ACY, $2 \mu \mathrm{M})$ on caspase-3/7 activity (b) and intracellular ATP levels (c) in OPM-2 cells. d Anti-tumour activity of bortezomib (Bort) alone or in combination with C1A in HCT116 xenograft model. Both C1A and bortezomib were given intraperitoneally once daily (q.d.). e Corresponding body weights during the time course of the study. Results are representative of three independent experiments performed in triplicate (mean \pm SEM). NS, not significant compared to control. ${ }^{* P}<0.05,{ }^{* * P}$ $<0.005,{ }^{* * *} \mathrm{P}<0.0001$.

the proteasome/autophagosome to ride it of potentially toxic protein. Myc, an oncoprotein that is frequently genetically amplified or whose transcriptional regulation is aberrant, potently activates protein synthesis via eukaryotic initiation factor $4 \mathrm{~F}$ (elF4F), the key regulator of the mRNA-ribosome recruitment phase of translation initiation. ${ }^{39}$ Indeed, in multiple myeloma cells, Myc has been shown to critically regulate aggresome/autophagosome formation and apoptosis in response to bortezomib and SAHA. ${ }^{40}$ Recently, Belounis et al. ${ }^{21}$ showed that autophagy is present in neuroblastoma cells, induced by chemotherapy and associated with chemoresistance. These studies warrant assessment of HDAC6 inhibitors in Myc-dependent cancers including B cell lineage (C-Myc-driven) and neuroblastoma (N-Myc-driven).

Rat fibroblast cell lines with endogenous levels of Myc $(\mathrm{Myc}+/+)$, an isogenic line bearing a homozygous deletion of Myc $(M y c-/-)$, and Myc-l- cells stably transduced with a lentiviral vector encoding wild-type human Myc (Myc-/-wtMyc) showed differential C-Myc expression (Fig. 4a). ${ }^{24}$ HDAC6 expression (using rat HDAC6 antibody) was higher in Myc-/-wtMyc and $M y c+/+$ cells compared to Myc-/- cells (Fig. 4a).

LC3 expression, cell growth inhibition by C1A and C1A-induced caspase-3/7 activation all increased in the order Myc-/- cells $<$ $M y c+/+$ cells $<M y c-/-$ wtMyc, consistent with C-Myc-dependent autophagy and sensitivity to HDAC6 inhibition (Fig. 4b, c).

While these results suggests that Myc may play a role in sensitivity to HDAC6 inhibition, the exact mechanisms are still unclear. We therefore investigated the effects of $C 1 \mathrm{~A}$ and $\mathrm{ACY}$ 1215 on ER stress and Myc. Both inhibitors triggered limited increases in CHOP, ATF4 and P58IPK mRNA-components of the UPR and thus indicators of ER stress-at $24 \mathrm{~h}$ in Myc high KELLY cells, suggesting that the changes in caspase- $3 / 7$ seen at this time point were largely independent of ER stress (Fig. 5a). A concentration-dependent induction of CHOP, ATF4 and P58IPK mRNA expression at $72 \mathrm{~h}$ demonstrate that C1A and ACY-1215 caused a late-onset transcriptional ER stress response. However, we could not detect increased phosphorylation of elF $2 a^{\text {Ser51 }}$ (Fig. 5b), an upstream biomarker of UPR activation, early $(24 \mathrm{~h})$ or late (48-72 h) after C1A or ACY-1215 treatment. In fact, C1A and ACY-1215 treatment resulted in decreased elF2a phosphorylation in KELLY cells and in Tet21/N cells. In the latter cells, the reduction in elF2a phosphorylation appeared to be largely independent of whether N-Myc was expressed at low (+Dox) or high (-Dox) levels. Thus, C1A and ACY-1215 induce an atypical and N-Mycindependent late-onset ER stress response. Interestingly, N-Myc mRNA expression was increased by $\mathrm{C} 1 \mathrm{~A}$, whereas $\mathrm{N}-\mathrm{Myc}$ protein levels were virtually undetectable in KELLY cells upon C1A treatment $(1$ and $10 \mu \mathrm{M})$ and in Myc -low Tet $21 / \mathrm{N}$ cells $(10 \mu \mathrm{M}$ C1A; Fig. 5b). We also observed that C1A led to a reversal in the LC3B-II/I ratio in KELLY cells, with an overall increase in detectable LC3B-II/I on immunoblots. In Tet21/N cells, the effects of $10 \mu \mathrm{M}$ C1A on LC3B-II/I were comparable in N-Myc high and low cells, but appeared different at the lower dose of $1 \mu \mathrm{M}$ (Fig. 5c). Taken together, C1A downregulates N-Myc protein levels despite persistent transcriptional induction, and its effects on autophagic processes appear to be partly linked to N-Myc expression. The precise mechanisms by which $\mathrm{C} 1 \mathrm{~A}$ treatment regulates $\mathrm{N}-\mathrm{Myc}$, 
a

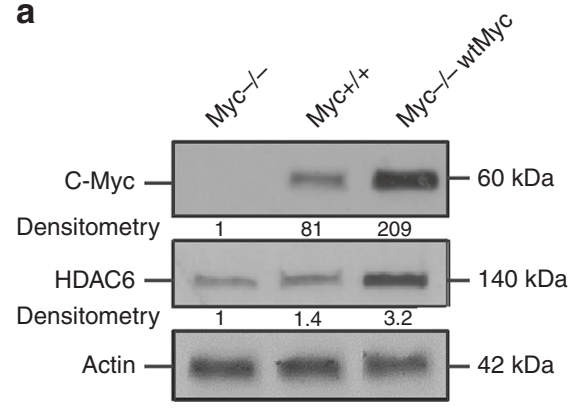

b

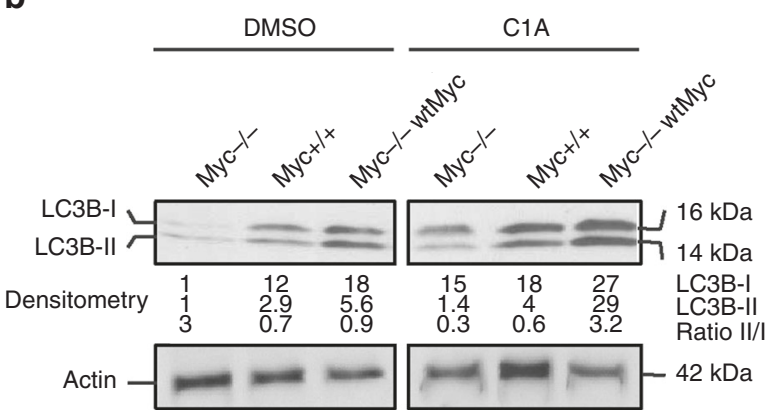

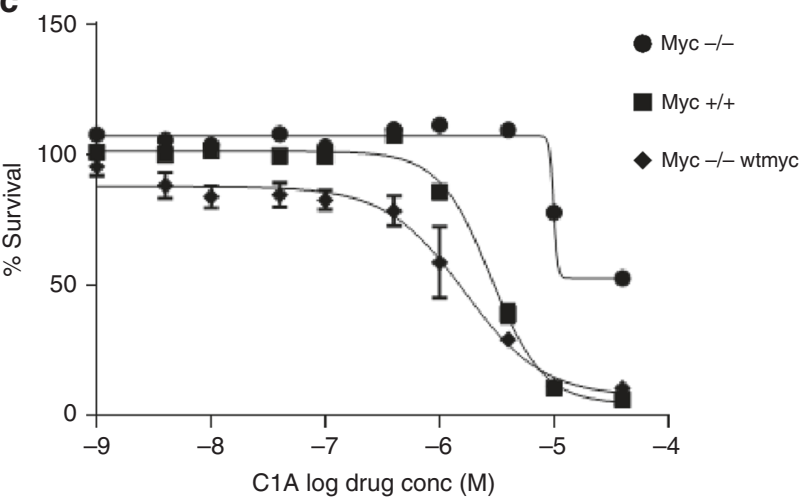

d

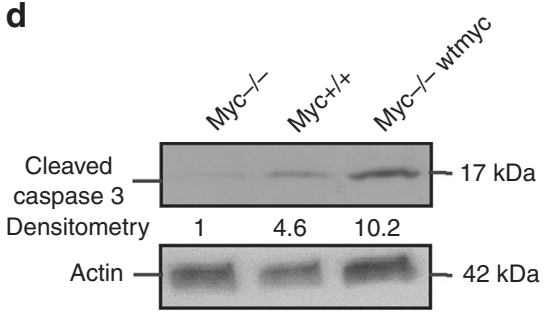

Fig. 4 Reduced expression of Myc abrogates the ability of HDAC6i to resolve autophagic response in TGR-1 rat fibroblasts. A cell line with endogenous levels of Myc. (Myc+/+), an isogenic line bearing a homozygous deletion of Myc (Myc-/ - ), and Myc-/ - cells stably transduced with a lentiviral vector encoding wild-type human Myc (Myc-/-wtMyc cells) were used in this study. a Western blot showing relative levels of HDAC6 in cell lines with different Myc expression. b Relative expression of LC3 following treatment with C1A (10 $\mu$ M) or DMSO for $24 \mathrm{~h}$. c Growth inhibitory effect of C1A over $72 \mathrm{~h}$ of treatment. Results are expressed as a percentage of control cells. $\mathbf{d}$ Impact of C1A following $24 \mathrm{~h}$ treatment with $\mathrm{C} 1 \mathrm{~A}$ at $10 \mu \mathrm{M}$ on cleaved caspase-3, as a marker of apoptosis

and how their activity relates to autophagy and the UPR, remain to be established but are likely to be biologically relevant.

HDAC6-selective inhibitors C1A and ACY-1215 were potent in multiple myeloma cell lines (ARH77, JJN3, KMS12, U266, RPMI8226, KMS11, OPM-2) with a mean $\mathrm{GI}_{50}$ of 0.48 and 1.04, respectively (Supplementary Table 1 ). In neuroblastoma cells (KELLY, SH-SY5Y, SHEP, SKNAS, SK-N-BE(2)C, IMR32, SKNSH), the $\mathrm{Gl}_{50}$ for $\mathrm{C} 1 \mathrm{~A}$ ranged between 0.18 and 16; with SAHA, the range was narrower (0.35 and 1.4) (Supplementary Table 1). From previous reports, we could classify neuroblastoma cell lines with no or low N-Myc expression (SHEP, SKNAS), with N-Myc amplification (IMR32, SK-N-BE(2)C and SH-SY5Y) and with high $\mathrm{N}$-Myc amplification (KELLY). ${ }^{41-44}$ Sensitivity of the neuroblastoma cell lines to C1A was found to be dependent on N-Myc protein expression, with KELLY being the most sensitive cell line (Fig. 6a). Using tubastatin A as a positive control, we showed that caspase-3/7 activation following HDAC6-selective C1A treatment of two neuroblastoma cell lines was inhibitor type and concentration dependent (Fig. 6b). Given the sensitivity of Mycpositive cells to $\mathrm{C} 1 \mathrm{~A}$, we correlated $\mathrm{C} 1 \mathrm{~A}$ sensitivity in the $\mathrm{NCl60}$ cell line panel to Myc expression. There was no correlation between C1A sensitivity and C-Myc or N-Myc mRNA expression (Supplementary Fig. 3), indicating that Myc mRNA expression per se does not represent a robust biomarker of C1A sensitivity ordinarily or in the context of autophagy.

From the sensitivity screen and N-Myc protein expression data, we selected the KELLY neuroblastoma cell line for further testing in vivo. Daily injection of single agent $\mathrm{C} 1 \mathrm{~A}$ at $20 \mathrm{mg} / \mathrm{kg}$ b.i.d. led to profound tumour growth arrest in the KELLY xenografts without any effect on mouse body weight (Fig. 6c, d).

\section{DISCUSSION}

This study shows that the hydroxamate-based small-molecule $\mathrm{C} 1 \mathrm{~A}$ can phenocopy HDAC6 inhibition and modulate autophagy in cancer cells from different origins. Markers of autophagy (i.e., LC3 and p62) were indeed altered following treatment in colon cancer and osteosarcoma cells. Not discounting other mechanisms of cell death, C1A was shown to induce apoptosis in those cells in the same range of concentrations and it is possible that autophagy served as a survival mechanism that when abrogated led to cell death. Markers of autophagy (e.g. the increased expressions of LC3BII and LC3BI in HCT116), however, suggested a blockade of the autophagy mechanism rather than an increase in the flux. We have also demonstrated in MEFs that C1A could phenocopy HDAC6 genetic knockout and alter the fusion of the autophagosomes and lysosomes. Autophagy has indeed been originally considered to be a non-specific bulk pathway by which cells scavenge cytoplasmic proteins and organelles in response to starvation. ${ }^{45}$ Other extracellular or intracellular stress signals such as growth factor deprivation, ER stress and pathogen infection were also shown to induce autophagy. ${ }^{46}$ The effects of $\mathrm{C} 1 \mathrm{~A}$ on apoptosis may be ER stress unrelated. C1A could potentially be used to modulate apoptosis and ER stress, and further applications are currently under investigation. Targeting protein homeostasis, both proteasomal and autophagy pathways were previously used as a strategy to treat nonsolid tumours. Hideshima et al. ${ }^{47}$ paved the way by using tubacin and bortezomib in pre-clinical models of multiple myeloma. HDAC6 inhibitor ACY-1215 has progressed to phase I/II clinical trials, but was associated with unfavourable pharmacokinetic properties. ${ }^{17,38}$ This outcome was potentially resolved with orally bioavailable compound WT161 that showed 
a

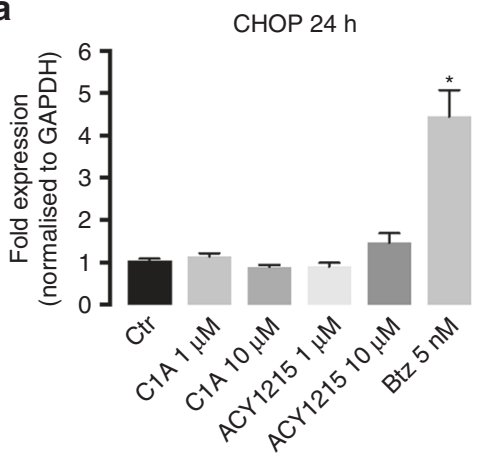

CHOP $72 \mathrm{~h}$

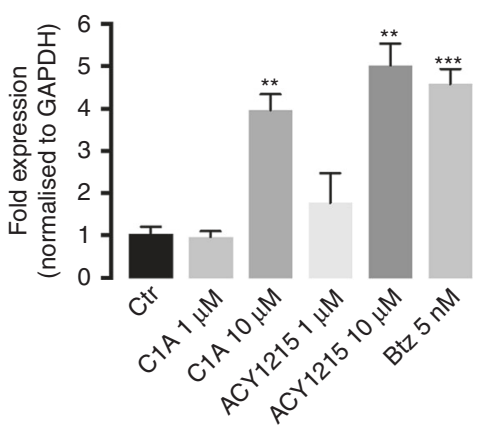

ATF4 $24 \mathrm{~h}$

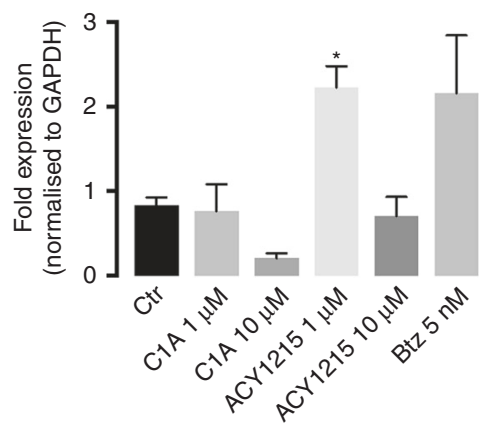

ATF4 $72 \mathrm{~h}$

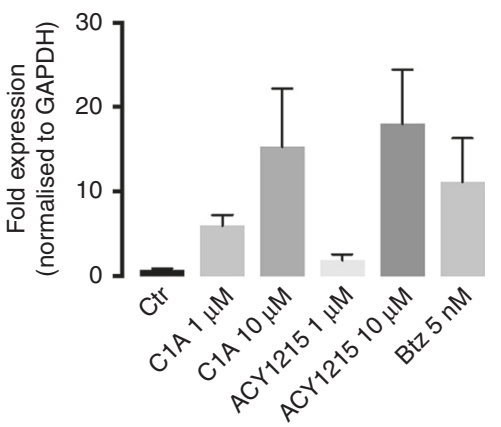

P58IPK $24 \mathrm{~h}$

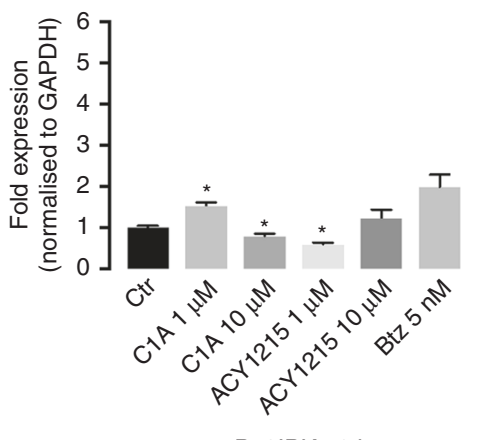

P58IPK $72 \mathrm{~h}$

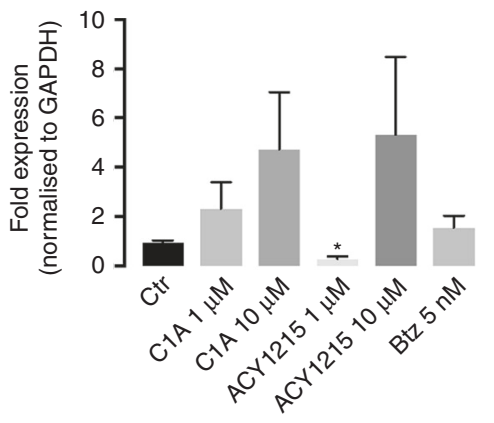

b
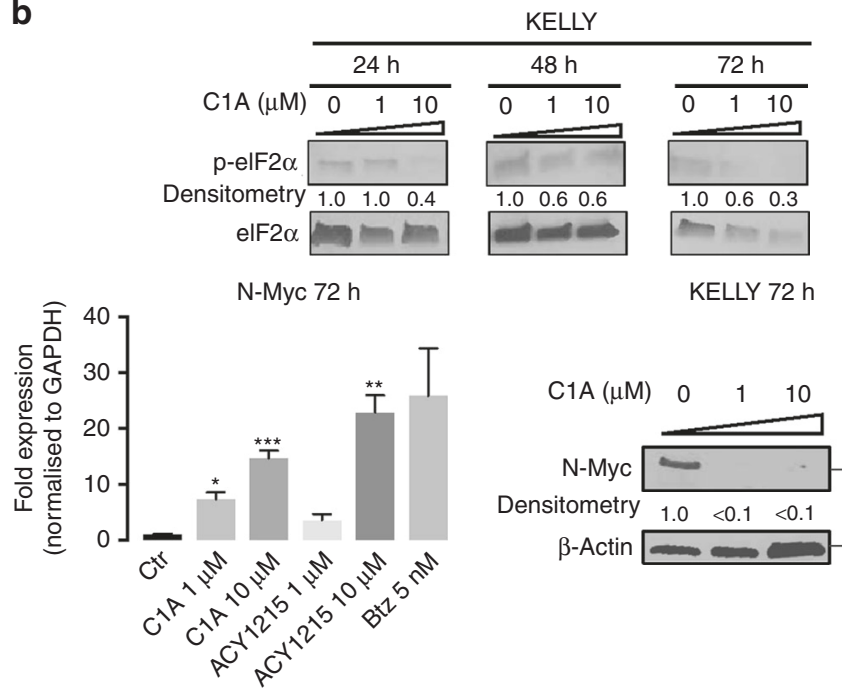

KELLY $72 \mathrm{~h}$

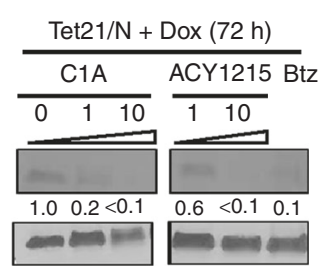

Tet21/N - Dox (72 h)

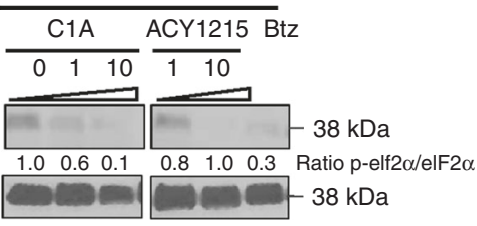

Tet21/N $72 \mathrm{~h}$

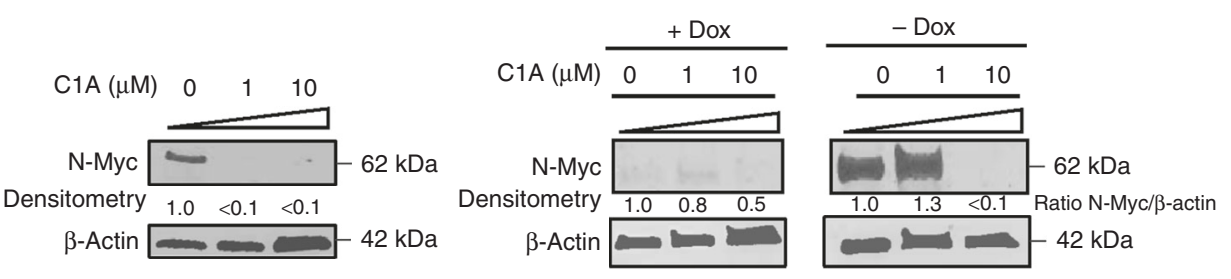

C

KELLY $72 \mathrm{~h}$
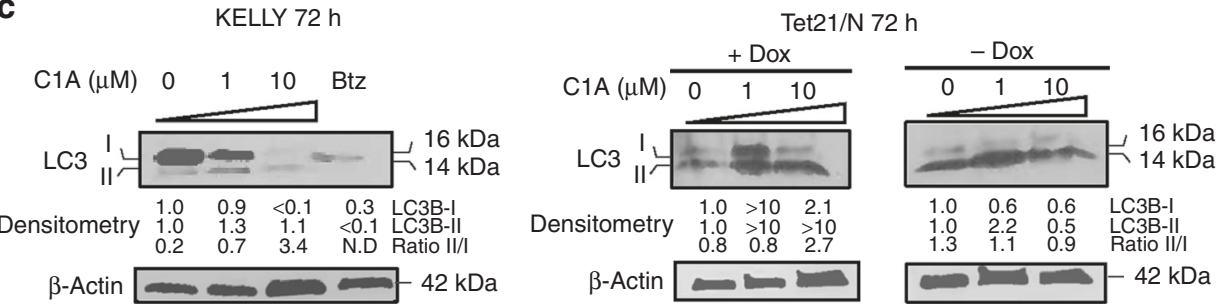

Fig. 5 Effect of HDAC6 inhibitors on ER stress and protein recycling in N-Myc high and low cells. a Effect of HDAC6 inhibitors on ER stress. NMyc high KELLY cells were treated continuously with vehicle, C1A $(1,10 \mu \mathrm{M})$, ACY-1215 (1, $10 \mu \mathrm{M})$ or bortezomib (Btz, $5 \mathrm{nM})$ for 24 (acute response) or $72 \mathrm{~h}$ (chronic response) and samples were analysed by real-time quantitative PCR for CHOP, ATF4 and P58IPK mRNA. b Effect of HDAC6 inhibitors on p-elF2 $\alpha /$ elF2 $\alpha$ protein expression (top panel) and N-Myc mRNA or protein expression (lower panel) in KELLY cells or in NMyc-high Tet21/N (-Dox) and N-Myc-low Tet21/N cells (+Dox) cells. The high cell death observed with C1A-treated KELLY cells at $72 \mathrm{~h}$ may account for the inconsistent density of elF $2 \alpha$ seen across the stated concentrations. Densitometry of ratios p-elF $2 \alpha /$ elF $2 \alpha$ and N-Myc/ $\beta$-actin of the treated groups were normalised to the untreated at respective time points. Dox, $2 \mu \mathrm{g} / \mathrm{mL}$ doxycycline. c Effect of HDAC6 inhibitors on LC3I and LC3-II proteins. ${ }^{\ddagger}$ Note that absolute LC3-II/LC3I ratios are presented. Dox, $2 \mu \mathrm{g} / \mathrm{mL}$ doxycycline. Results are representative of three independent experiments performed in triplicate (mean \pm SEM). NS, not significant compared to control. ${ }^{*} P<0.05,{ }^{* * P}<0.005,{ }^{* * * P}<0.0001$. 


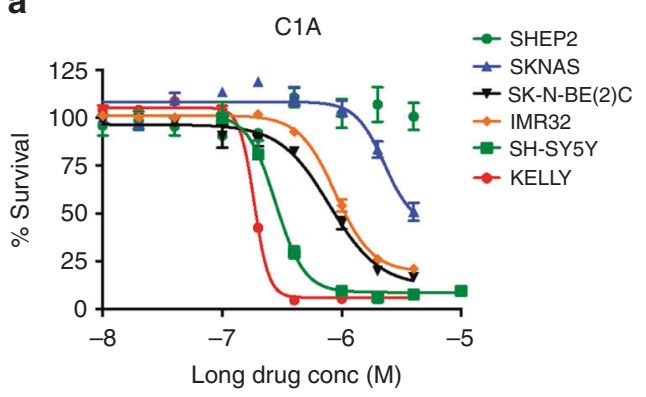

C

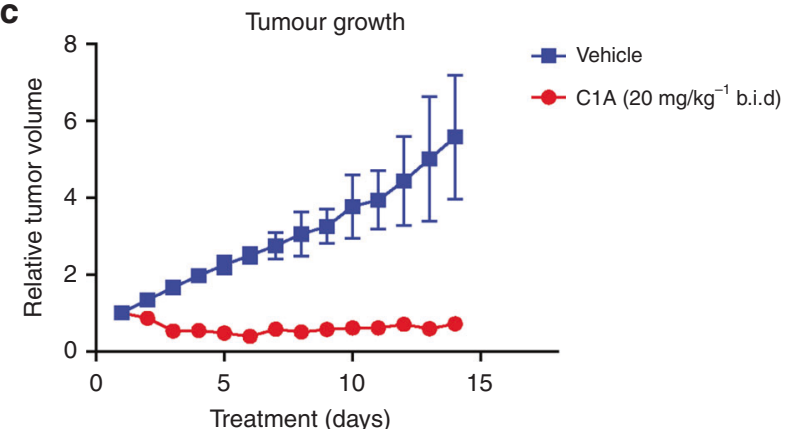

b

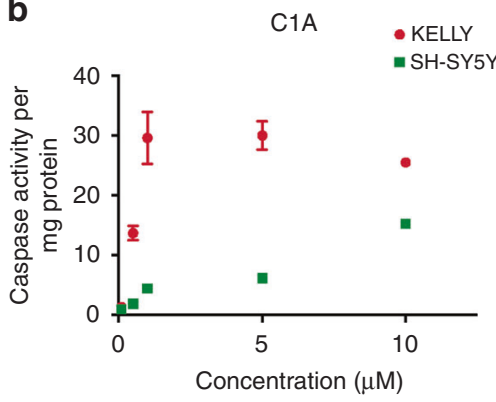

d

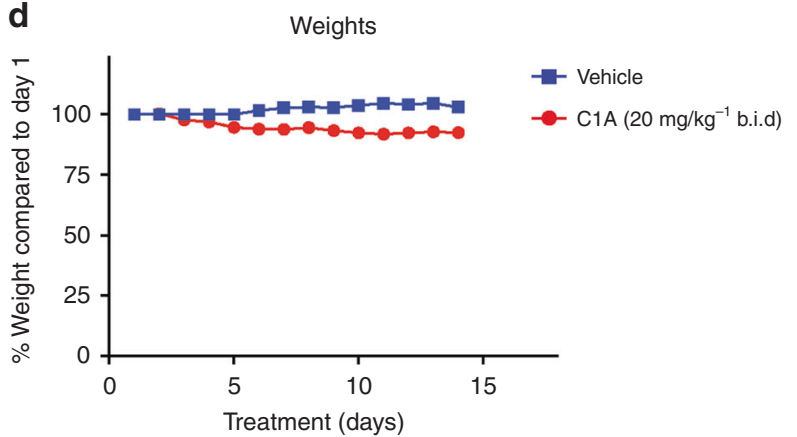

Fig. 6 Myc-proficient neuroblastoma cells are sensitive to C1A. a Growth inhibitory effect of C1A in a panel of neuroblastoma with different NMyc expression. $\mathbf{b}$ Impact of HDAC6 inhibitors, C1A and tubastatin A, on caspase- 3/7 activity in SH-SY5Y and KELLY cells. Data are normalised by the amount of protein. Cells were treated for $24 \mathrm{~h}$ at the indicated concentration. c, d In vivo anti-tumour activity (c) of C1A and corresponding body weights (d) in KELLY mouse model of neuroblastoma. C1A was given twice daily at $20 \mathrm{mg} / \mathrm{kg}$ for 14 days $(n=3)$

promise in combination with bortezomib in a pre-clinical model of multiple myeloma. ${ }^{17}$ To our knowledge, our study is the first to describe a strategy of tackling protein homeostasis in order to treat solid tumours. C1A synergised with bortezomib in colon cancer cells, greatly enhancing caspase-3/7 activation and was able to reduce tumour growth in mouse models of disease.

We have shown that tumour types, with high protein turnover in general could be more susceptible to therapy with HDAC6 inhibitors. We have exemplified our findings with KRAS-positive colon cancer cells, Myc-amplified neuroblastoma (N-Myc-driven) and confirmed sensitivity of combination therapy in B cell lineage (C-Myc-driven). We were however not able to correlate sensitivity of C1A more broadly in the $\mathrm{NCl} 60$ panel of cell lines with C-Myc or $\mathrm{N}$-Myc expression. Other than lineage dependence, it is possible that expression of Myc per se may be insufficient to predict sensitivity to HDAC6 inhibitors in all cell types. Myc transcriptional activity may lie in its ability to couple with binding partners (e.g. MAX) and form functional complexes. ${ }^{48}$ Myc activity can also be antagonised by the presence of MAD1 that acts as a transcriptional repressor. ${ }^{49}$ Upstream regulators could also affect the activity of Myc. For instance, RAS mutation was shown to contribute to the oncogenic role of N-Myc. ${ }^{50}$ The tyrosine kinase SRC has also been implicated in mediating growth factor signal-induced C-Myc expression. $^{51,52}$ Even though a complex scenario likely exists for generation of superfluous protein requiring degradation-with Myc being a player-we can speculate that tumours would be primed to HDAC6 inhibitors as demonstrated in this report. ${ }^{53}$

We observed an elevated HDAC6 expression in fibroblasts overexpressing C-Myc, which is consistent with previous reports. For instance, Nawrocki et al. ${ }^{40}$ have shown, in human foreskin fibroblasts transfected with Myc, increased protein synthesis and aggresome/autophagosome formation alongside increased HDAC6 expression, suggesting that further that HDAC6 is a direct Myc transcriptional target, ${ }^{40}$ although our current work in neuroblastoma cells showed that $\mathrm{C} 1 \mathrm{~A}$ increased Myc mRNA level and decreased its expression level. Another report by Lwin et al. ${ }^{54}$ linked C-Myc and
HDAC6 expression in cell lines and primary lymphoma samples of mantle cell lymphomas and other B cell lymphomas. The authors provided evidence that activation of HDAC6 may be modulated by the microenvironment. This suggests that HDAC6 expression could also be predictive of sensitivity to HDAC6 inhibitors, but we previously showed no correlation between sensitivity to $\mathrm{C} 1 \mathrm{~A}$ and HDAC6 expression in the $\mathrm{NCl} 60$ panel. ${ }^{19}$ This might not seem surprising as the cells were tested under 'basic' conditions, which emphasises the necessity to develop and test more refined in vitro and primary tumour models that would mimic more closely an in vivo microenvironment. ${ }^{55}$ Such refinement would help ascertain the influence of Myc activity on HDAC6 expression, and further, prediction of HDAC6 inhibitors' sensitivity in numerous tumour types.

To conclude, we have shown that pharmacological inhibition of HDAC6 by C1A resolves autophagy induced by 3-MA and is cytotoxic to tumour cells alone or in combination with a proteasome inhibitor. We showed that the combination is more efficacious in vivo in nonsolid and solid tumours, warranting its use for in vivo pre-clinical autophagy research. Finally, we identified Myc expression (in neuroblastoma) as worthy of further evaluation as a predictive marker of response.

\section{ACKNOWLEDGEMENTS}

Several cell lines were kindly provided by our collaborators. Their origins are described in Materials and methods section.

\section{AUTHOR CONTRIBUTIONS}

M.K. designed and performed experiments and wrote the manuscript. E.v.H., Y.L., H.A., and K.A. performed experiments and edited the manuscript. H.W.A. and E.O.A. designed and edited the manuscript.

\section{ADDITIONAL INFORMATION}

Supplementary information is available for this paper at https://doi.org/10.1038/ s41416-018-0232-5. 
Competing interests: The authors declare no competing interests.

Availability of data and material: All datasets on which the conclusions of the paper rely will be available to readers. Materials could be requested by email.

Ethics approval and consent to participate: No human tissue was used in this study. All animal experiments were done by licensed investigators in accordance with the United Kingdom Home Office Guidance on the Operation of the Animal (Scientific Procedures) Act 1986 (HMSO, London, UK, 1990) and within guidelines set out by the United Kingdom National Cancer Research Institute Committee on Welfare of Animals in Cancer Research. ${ }^{28}$ Consent for publication No individual person's data were used in this study.Funding This study was supported by CR-UK and EPSRC Cancer Imaging Centre at Imperial College London, in association with the MRC and Department of Health (England) under grant C2536/A10337 and CR-UK under grant C2536/A16584.

\section{REFERENCES}

1. White, E. Deconvoluting the context-dependent role for autophagy in cancer. Nat. Rev. Cancer 12, 401-410 (2012).

2. Mathew, R., Karantza-Wadsworth, V. \& White, E. Role of autophagy in cancer. Nat. Rev. Cancer 7, 961-967 (2007).

3. Lambert, L. A. et al. Autophagy: a novel mechanism of synergistic cytotoxicity between doxorubicin and roscovitine in a sarcoma model. Cancer Res. 68, 7966-7974 (2008).

4. Sui, X. et al. Autophagy and chemotherapy resistance: a promising therapeutic target for cancer treatment. Cell Death Dis. 4, e838 (2013).

5. Auner, H. W. \& Cenci, S. Recent advances and future directions in targeting the secretory apparatus in multiple myeloma. Br. J. Haematol. 168, 14-25 (2015).

6. Verfaillie, T., Salazar, M., Velasco, G. \& Agostinis, P. Linking ER stress to autophagy: potential implications for cancer therapy. Int. J. Cell Biol. 2010, 930509 (2010).

7. Poklepovic, A. \& Gewirtz, D. A. Outcome of early clinical trials of the combination of hydroxychloroquine with chemotherapy in cancer. Autophagy 10, 1478-1480 (2014).

8. Rosenfeld, M. R. et al. A phase I/II trial of hydroxychloroquine in conjunction with radiation therapy and concurrent and adjuvant temozolomide in patients with newly diagnosed glioblastoma multiforme. Autophagy 10, 1359-1368 (2014).

9. Boone, B. A. et al. Safety and biologic response of pre-operative autophagy inhibition in combination with gemcitabine in patients with pancreatic adenocarcinoma. Ann. Surg. Oncol. 22, 4402-4410 (2015).

10. Wu, Y. et al. Synthesis and screening of 3-MA derivatives for autophagy inhibitors. Autophagy 9, 595-603 (2013).

11. Yao, T. P. The role of ubiquitin in autophagy-dependent protein aggregate processing. Genes Cancer 1, 779-786 (2010).

12. Watanabe, Y. \& Tanaka, M. p62/SQSTM1 in autophagic clearance of a nonubiquitylated substrate. J. Cell Sci. 124(Part 16), 2692-2701 (2011).

13. Kawaguchi, Y. et al. The deacetylase HDAC6 regulates aggresome formation and cell viability in response to misfolded protein stress. Cell 115, 727-738 (2003).

14. Iwata, A., Riley, B. E., Johnston, J. A. \& Kopito, R. R. HDAC6 and microtubules are required for autophagic degradation of aggregated huntingtin. J. Biol. Chem. 280, 40282-40292 (2005).

15. Ouyang, $H$. et al. Protein aggregates are recruited to aggresome by histone deacetylase 6 via unanchored ubiquitin C termini. J. Biol. Chem. 287, 2317-2327 (2012).

16. Dimopoulos, M. et al. Vorinostat or placebo in combination with bortezomib in patients with multiple myeloma (VANTAGE 088): a multicentre, randomised, double-blind study. Lancet Oncol. 14, 1129-1140 (2013).

17. Hideshima, T. et al. Discovery of selective small-molecule HDAC6 inhibitor for overcoming proteasome inhibitor resistance in multiple myeloma. Proc. Natl. Acad. Sci. USA 113, 13162-13167 (2016).

18. Lee, J. H. et al. Development of a histone deacetylase 6 inhibitor and its biological effects. Proc. Natl. Acad. Sci. USA 110, 15704-15709 (2013).

19. Kaliszczak, M. et al. A novel small-molecule hydroxamate preferentially inhibits HDAC6 activity and tumour growth. Br. J. Cancer 108, 342-350 (2013).

20. Dong, L. H. et al. Histone deacetylase inhibitor potentiated the ability of MTOR inhibitor to induce autophagic cell death in Burkitt leukemia/lymphoma. J. Hematol. Oncol. 6, 53 (2013).

21. Belounis, A. et al. Autophagy is associated with chemoresistance in neuroblastoma. BMC Cancer 16, 891 (2016).

22. Kaliszczak, M., Trousil, S., Ali, T. \& Aboagye, E. O. AKT activation controls cell survival in response to HDAC6 inhibition. Cell Death Dis. 7, e2286 (2016).
23. Lutz, W. et al. Conditional expression of N-myc in human neuroblastoma cells increases expression of alpha-prothymosin and ornithine decarboxylase and accelerates progression into S-phase early after mitogenic stimulation of quiescent cells. Oncogene 13, 803-812 (1996).

24. Graves, J. A. et al. Mitochondrial structure, function and dynamics are temporally controlled by c-Myc. PLoS ONE 7, e37699 (2012).

25. Kaliszczak, M. et al. Optimization of the antitumor activity of sequence-specific pyrrolobenzodiazepine derivatives based on their affinity for $A B C$ transporters. AAPS J. 12, 617-627 (2010).

26. Parzych, K. et al. Inadequate fine-tuning of protein synthesis and failure of amino acid homeostasis following inhibition of the ATPase VCP/p97. Cell Death Dis. 6, e2031 (2015)

27. Vichai, V. \& Kirtikara, K. Sulforhodamine B colorimetric assay for cytotoxicity screening. Nat. Protoc. 1, 1112-1116 (2006).

28. Workman, P. et al. Guidelines for the welfare and use of animals in cancer research. Br. J. Cancer 102, 1555-1577 (2010).

29. Kaliszczak, M. et al. Development of a cyclin-dependent kinase inhibitor devoid of ABC transporter-dependent drug resistance. Br. J. Cancer 109, 2356-2367 (2013).

30. Krypuy, M., Newnham, G. M., Thomas, D. M., Conron, M. \& Dobrovic, A. High resolution melting analysis for the rapid and sensitive detection of mutations in clinical samples: KRAS codon 12 and 13 mutations in non-small cell lung cancer. BMC Cancer 6, 295 (2006).

31. Simi, L. et al. High-resolution melting analysis for rapid detection of KRAS, BRAF, and PIK3CA gene mutations in colorectal cancer. Am. J. Clin. Pathol. 130, 247-253 (2008).

32. Mizushima, N., Yoshimorim, T. \& Levine, B. Methods in mammalian autophagy research. Cell 140, 313-326 (2010).

33. Zhang X-j, ChenS. \& Huang K-x, Le. W.-d Why should autophagic flux be assessed? Acta Pharmacol. Sin. 34, 595-599 (2013).

34. Nakaso, K. et al. Transcriptional activation of p62/A170/ZIP during the formation of the aggregates: possible mechanisms and the role in Lewy body formation in Parkinson's disease. Brain Res. 1012, 42-51 (2004).

35. Ganley, I. G., Wong, P. M., Gammoh, N. \& Jiang, X. Distinct autophagosomal-lysosomal fusion mechanism revealed by thapsigargin-induced autophagy arrest. Mol. Cell 42, 731-743 (2011).

36. Pitts, T. M., Morrow, M., Kaufman, S. A., Tentler, J. J. \& Eckhardt, S. G. Vorinostat and bortezomib exert synergistic antiproliferative and proapoptotic effects in colon cancer cell models. Mol. Cancer Ther. 8, 342-349 (2009).

37. Santo, L. et al. Preclinical activity, pharmacodynamic, and pharmacokinetic properties of a selective HDAC6 inhibitor, ACY-1215, in combination with bortezomib in multiple myeloma. Blood 119, 2579-2589 (2012).

38. Vogl, D. T. et al. Ricolinostat, the first selective histone deacetylase 6 inhibitor, in combination with bortezomib and dexamethasone for relapsed or refractory multiple myeloma. Clin. Cancer Res. 23, 3307-3315 (2017).

39. Nesbit, C. E., Tersak, J. M. \& Prochownik, E. V. MYC oncogenes and human neoplastic disease. Oncogene 18, 3004-3016 (1999).

40. Nawrocki, S. T. et al. Myc regulates aggresome formation, the induction of Noxa, and apoptosis in response to the combination of bortezomib and SAHA. Blood 112, 2917-2926 (2008).

41. Chesler, L. et al. Chemotherapy-induced apoptosis in a transgenic model of neuroblastoma proceeds through p53 induction. Neoplasia 10, 1268-1274 (2008).

42. Chesler, L. et al. Inhibition of phosphatidylinositol 3-kinase destabilizes Mycn protein and blocks malignant progression in neuroblastoma. Cancer Res. 66, 8139-8146 (2006).

43. Dolman, M. E. et al. Cyclin-dependent kinase inhibitor AT7519 as a potential drug for MYCN-dependent neuroblastoma. Clin. Cancer Res. 21, 5100-5109 (2015).

44. Huang, R. et al. MYCN and MYC regulate tumor proliferation and tumorigenesis directly through BMI1 in human neuroblastomas. FASEB J. 25, 4138-4149 (2011).

45. Kamada, Y., Sekito, T. \& Ohsumi, Y. Autophagy in yeast: a TOR-mediated response to nutrient starvation. Curr. Top. Microbiol. Immunol. 279, 73-84 (2004).

46. He, C. \& Klionsky, D. J. Regulation mechanisms and signaling pathways of autophagy. Annu. Rev. Genet. 43, 67-93 (2009).

47. Hideshima, T. et al. Small-molecule inhibition of proteasome and aggresome function induces synergistic antitumor activity in multiple myeloma. Proc. Natl. Acad. Sci. USA 102, 8567-8572 (2005).

48. Grandori, C., Cowley, S. M., James, L. P. \& Eisenman, R. N. The Myc/Max/Mad network and the transcriptional control of cell behavior. Annu. Rev. Cell Dev. Biol. 16, 653-699 (2000).

49. Luscher, B. MAD1 and its life as a MYC antagonist: an update. Eur. J. Cell Biol. 91, 506-514 (2012) 
The HDAC6 inhibitor C1A modulates autophagy substrates in diverse cancer... M Kaliszczak et al.

50. Schwab, M., Varmus, H. E. \& Bishop, J. M. Human N-myc gene contributes to neoplastic transformation of mammalian cells in culture. Nature 316, 160-162 (1985).

51. Barone, M. V. \& Courtneidge, S. A. Myc but not Fos rescue of PDGF signalling block caused by kinase-inactive Src. Nature 378, 509-512 (1995).

52. Blake, R. A. et al. SU6656, a selective src family kinase inhibitor, used to probe growth factor signaling. Mol. Cell. Biol. 20, 9018-9027 (2000).

53. Furstoss, O. et al. c-Abl is an effector of Src for growth factor-induced c-myc expression and DNA synthesis. EMBO J. 21, 514-524 (2002).

54. Lwin, T. et al. A microenvironment-mediated c-Myc/miR-548m/HDAC6 amplification loop in non-Hodgkin B cell lymphomas. J. Clin. Invest. 123, 4612-4626 (2013).

55. Gillet, J. P. et al. Redefining the relevance of established cancer cell lines to the study of mechanisms of clinical anti-cancer drug resistance. Proc. Natl. Acad. Sci. USA 108, 18708-18713 (2011).
Open Access This article is licensed under a Creative Commons Cc) Attribution 4.0 International License, which permits use, sharing,
adaptation, distribution and reproduction in any medium or format, as long as you give appropriate credit to the original author(s) and the source, provide a link to the Creative Commons license, and indicate if changes were made. The images or other third party material in this article are included in the article's Creative Commons license, unless indicated otherwise in a credit line to the material. If material is not included in the article's Creative Commons license and your intended use is not permitted by statutory regulation or exceeds the permitted use, you will need to obtain permission directly from the copyright holder. To view a copy of this license, visit http://creativecommons. org/licenses/by/4.0/.

(c) The Author(s) 2018 